\title{
Unpreventable maternal death despite the advances in antenatal care
}

\author{
Warushahennadi $\mathbf{J}^{1}$, Kularathne $\mathrm{SK}^{2}$, Ranawaka $\mathbf{N}^{3}$ \\ Departments of ${ }^{1}$ Forensic Medicine, ${ }^{2}$ Obstretics \& Gynaecology, ${ }^{3}$ Pathology, \\ Faculty of Medicine, University of Ruhuna, Galle, Sri Lanka.
}

Correspondence: Dr. Janaki Warushahennadi

e-mail: janakiwh@gmail.com

https://orcid.org/0000-0002-6880-5513

\section{Introduction}

The annual incidence of aortic dissection (AoD) during pregnancy is about 0.4 cases per 100,000 persons (1). Although it is a rare entity, aortic dissection in pregnancy may result in adverse maternal and fetal outcomes. Half of the aortic dissections occur in the third trimester of pregnancy and in the postpartum period due to the hyperdynaemic state and the hormonal effect on the vasculature $(2,3)$. Atherosclerosis, hypertension, genetic disorders of connective tissue such as Marfan's syndrome, genetic conditions such as Turner's syndrome, valvular abnormalities of the heart such as bicuspid aortic valve and vascular conditions like preeclampsia and cocaine abuse are some of the risk factors for AoD (4). The pregnancy alone with no underlying preexisting risk factors is a risk factor for AoD (5). The hyperdynamic changes in pregnancy and the increased oestrogen level which suppresses the synthesis of collagen and elastin may have weakened the vascular wall thereby playing an important role in the development of AoD during pregnancy (5).

Although it is difficult to diagnose the AoD, the measurement of diameter of the root of the aorta is a diagnostic criterion to identify the risk of acute AoD. The aortic root diameter more than $45 \mathrm{~mm}$ in pregnancy has a high risk for $\mathrm{AoD}(6)$.

\section{Case presentation}

A 28-year-old, 38-week primi was referred to a tertiary care hospital following sudden onset of burning epigastric pain which developed while returning from the routine visit to the cardiologist.
She was admitted to the hospital around 30 minutes after developing symptoms. She was treated for gastritis in the ward and a few hours later, she developed shortness of breath, tight chest pain and tachycardia. Emergency Lower Segment Caesarian Section (LSCS) was done and a live, nonasphyxiated baby was delivered. Soon after the extubation, she developed a sudden cardiac arrest and despite the intensive resuscitation measures, which include transfusion of six units of blood, she succumbed to injuries.

She was diagnosed with mild aortic regurgitation (AR) and trivial mitral regurgitation (MR) before pregnancy and was referred to the cardiologist during the first trimester and was confirmed with having a low cardiac risk. She was seen by the cardiologist during all three trimesters and was concluded to have low cardiac risk. During the last visit to the cardiologist which was approximately 30 minutes before admission to the hospital, she had undergone a 2D ECHO that has also revealed a low cardiac risk. She was not a known hypertensive and there was no history of connective tissue disorders.

The autopsy revealed $2750 \mathrm{ml}$ of blood in the combined chest cavity and $750 \mathrm{ml}$ of blood in the abdominal cavity. There was a collection of blood which was infiltrated into the mediastinal tissues surrounding the aorta. The collection of blood was present in the subintima of the aorta starting from the root of the aorta extending up to the ostia of the renal arteries in the abdominal aorta (Figure 1). The exact place of the dissection was unable to be determined at the autopsy. The abdominal cavity was normal except the postpartum appearance of the sectioned uterus. There was no bicuspid aortic valve. 
Several sections obtained from different sites of the aorta for histology revealed degeneration of tunica media and through and through micro-tears with leakage of blood into the surrounding tissues of the aorta (Figure 2). The cause of death was concluded as haemorrhagic shock following acute aortic dissection in a pregnant woman.

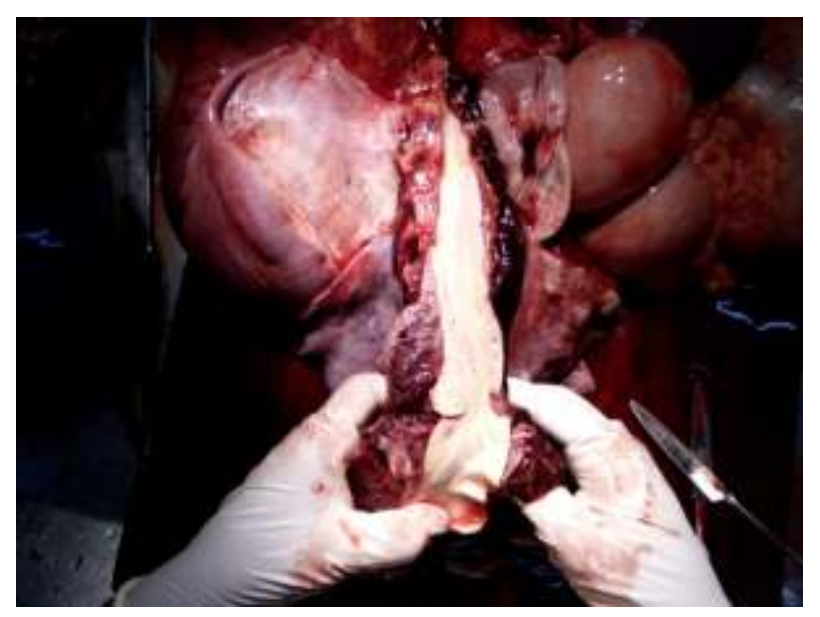

Figure 1: The eviscerated organs with the opened aorta. Note absence of tears on the intima.

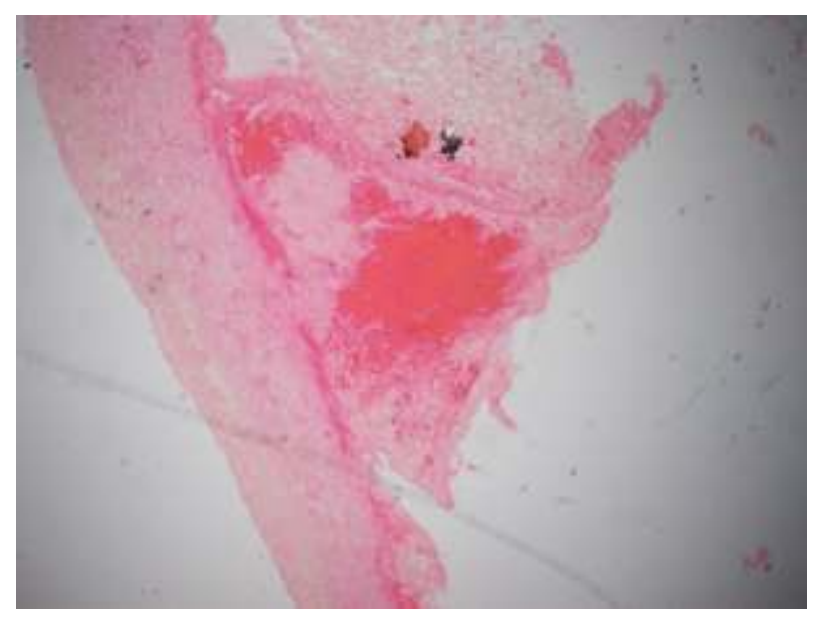

Figure 2: Micrograph of the aorta showing leakage of blood into its wall.

\section{Discussion}

This patient was diagnosed with AR and trivial MR which are not considered as risk factors for the aortic dissection. Among the valvular heart diseases, bicuspid aortic valve, which is a congenital anomaly of the aortic valve, is considered as a risk factor for acute AoD (7). This indicates that the deceased did not have a known preexisting risk factor for the acute AoD other than being pregnant. The hyperdynamic changes in pregnancy such as increased heart rate, stroke volume, cardiac output, and left ventricular dimensions may have affected on the forces on the aortic valve (6) and this may be exacerbated by increased outflow resistance in the distal aorta due to compression by the gravid uterus (5). The hormonal changes during pregnancy suppress the synthesis of collagen and elastin weakening the elastic fibers of the aortic vessel wall (8). Therefore these alterations during pregnancy may have caused the aortic dissection in the deceased.

The common symptoms at the onset of the AoD are, chest pain accounting for $12 \%$ and the back pain accounting for 55\% (9). Sudden onset of dyspnea, nausea and vomiting has been reported in a death due to AoD (10). Our patient had burning epigastric pain more suggestive of gastritis. The determination of having a low cardiac risk by the cardiologist a few hours before and the atypical clinical symptoms may have contributed to delay in suspecting AoD.

The evaluation by a cardiologist a few hours before death revealing a low cardiac risk was based on the measurements of the root of the aorta, which measured less than $40 \mathrm{~mm}$. For it to be high risk of AoD, the aortic root diameter should be more than $45 \mathrm{~mm}$ in pregnancy. However, thirty minutes later she developed symptoms and was admitted to the hospital. On suspicion of AoD, an emergency LSCS was performed. Pregnancy itself is a risk factor for the development of AoD, which can lead to sudden unexpected death. This case report highlights the fact that one cannot rely totally on investigation findings. Further, lack of reference values for aortic root diameter among Sri Lankans may have contributed to failure in early diagnosis. However, the increased risk of AoD during pregnancy should be kept in mind in patients with symptoms even without preexisting risk factors.

\section{References}

1. Thalmann M, Sodeck GH, Domanovits H, Gassberger M, Loewe $\mathrm{C}$, et al. Acute type A aortic dissection and pregnancy: a popular based study. Eur. J Cardiothorac Surg, 2011; 39(6): 159-63. 
2 Ozdemir B. Aortic Dissection in Pregnancy. Turkish Clinics J Int Med Sci, 2005; 1(50): 54-8.

3. Collins D. Aethiology and management of acute cardiac tamponade. Crit Care Resusc, 2004; 6: 54-8.

4. Nasiell J, Lindqvist PG. Aortic dissection in pregnancy: the incidence of a life threatening disease. Eur J Obstet Gynecol Reprod Biol 2010; 149: 120-21.

5. Shi-MinYuan. Aortic Dissection During Pregnancy: A Difficult Clinical Scenario: Review. Clini. Cardiol, 2013; 36(10): 576-84.

6. Immer FF, Bansi AG, Immer Bansi AS, et al. Aortic dissection in pregnancy : analysis of risk factors and outcome. Ann Thorac Surg. 2003: 76:309-314.
7. Nishadi AA, R Herath, G R C Silva, R Mohomead, P Wijesinghe, $S$ Padumadasa et al. A fatal case of dissecting aortic root aneurysm due to undiagnosed Marfan syndrome in the puerperium. Sri Lanka Journal of Obstetrics and Gynaecology, 2012;34(2): 55-7.

8. Ohlson L. Effects of the pregnant uterus on the abdominal aorta and its branches. Acta. Radiol Diagn. (Stockh) 1978: 19: $369-76$.

9. Fuster V, Andrews P. Medical treatment of the aorta. $L$. CardiolClin, 1999; 17(4): 697-715.

10. Kinney-Ham, H. Bryant Nguyen, Robert Steele, Elizebeth L Walters. Acute Aortic Dissection in Third Trimester Pregnancy without Risk Factors. West J Emerg Med, 2011 Nov; 12(4): 571-74. 